\title{
MALDI-MS Imaging of Features Smaller than the Size of the Laser Beam
}

\author{
John C. Jurchen, Stanislav S. Rubakhin, and Jonathan V. Sweedler \\ Department of Chemistry, University of Illinois, Urbana, Illinois, USA
}

\begin{abstract}
The feasibility of matrix-assisted laser desorption/ionization mass spectrometry (MALDI-MS) imaging of features smaller than the laser beam size has been demonstrated. The method involves the complete ablation of the MALDI matrix coating the sample at each sample position and moving the sample target a distance less than the diameter of the laser beam before repeating the process. In the limit of complete sample ablation, acquiring signal from adjacent positions spaced by distances smaller than the sample probe enhances image resolution as the measured analyte signal only arises from the overlap of the laser beam size and the non-ablated sample surface. Image acquisition of features smaller than the laser beam size has been demonstrated with peptide standards deposited on electron microscopy calibration grids and with neuropeptides originating from single cells. The presented MS imaging technique enables $\sim 25 \mu \mathrm{m}$ imaging spatial resolution using commercial MALDI mass spectrometers having irregular laser beam sizes of several hundred micron diameters. With appropriate sampling, the size of the laser beam is not a strict barrier to the attainable MALDI-MS imaging resolution. (J Am Soc Mass Spectrom 2005, 16, 1654-1659) (C) 2005 American Society for Mass Spectrometry
\end{abstract}

$\mathrm{T}$ he introduction of matrix assisted laser desorption/ionization (MALDI) [1, 2] and electrospray ionization (ESI) [3] has revolutionized the investigation of biological molecules by providing soft ionization methods linking biochemistry with the powerful analysis tools of mass spectrometry (MS). In the analysis of complex samples, such as biological tissue, MALDI is of particular interest because of its ability to desorb and ionize molecules of high molecular weight, such as proteins and peptides, providing excellent sensitivity while retaining considerable tolerance towards salts and other small molecules found at high concentration in tissue. It has been roughly 10 years since the first published applications in which MALDI-MS was used to create a chemical images of substrates [4,5]. The intervening decade has seen a considerable growth in techniques and instrumentation for MALDI-MS imaging, developed largely by Caprioli and coworkers [6-12] with contributions to sampling techniques [13-16] and instrumentation [17, 18] provided by others.

It is generally accepted that the maximal spatial imaging or profiling resolution of microprobe imaging techniques is determined by a combination of the size of the microprobe and the precision of the sample or microprobe positioning device. MALDI mass spectrometers typically use lasers having relatively large beam

Published online August 10, 2005

Address reprint requests to Professor J. V. Sweedler, Department of Chemistry, University of Illinois, 600 S. Mathews Ave. 63-5, Urbana, IL 61801, USA. E-mail: jsweedle@uiuc.edu sizes (about $100 \mu \mathrm{m}$ diameter) in the analysis of standard, dried-droplet preparations. Some effort has been put into decreasing the size of the laser beam sizes for MALDI-MS imaging and profiling of biological samples, particularly for samples containing a high proportion of peptidergic neurons or other secretory cells. Investigated biological samples of this nature include rat pituitary and rat pancreas [6], mouse brain and human brain tumor xenografts $[8,14]$, rat brain and rat brain tumors [9, 19], mouse epididymis [20], molluscan atrial gland [15], and molluscan peptidergic neurons [16].

Ideally, the spatial resolution of MALDI-MS imaging of analyte-rich tissues would approach the size of a single mammalian cell, 5 to $20 \mu \mathrm{m}$ in diameter. Several strategies have been used or suggested to decrease the laser beam diameter in imaging applications, including the placement of a pinhole aperture between the outlet of the laser and the focusing optics of the mass spectrometer $[6,13]$, decreasing the size of the fiber optic used to direct the laser into the MALDI source [9], and placing multiple lenses between the laser and the MALDI source [17]. Of particular note is the SMALDI platform developed in Spengler's laboratory [17], which is capable of producing a $<1 \mu \mathrm{m}$ on-target laser beam size and has a piezoelectric-driven translation stage capable of $0.25 \mu \mathrm{m}$ raster increments [17]. Another, more recent, innovation in high-resolution MALDI imaging is the development of an ion microscope that uses a large laser beam size $(\sim 200 \mu \mathrm{m})$ to generate ions simultaneously from an entire region of a sample and the impressive ion optics of a TRIFT II (triple focusing 
time-of-flight) mass spectrometer (Physical Electronics, Eden Prairie, MN) to maintain the relative spatial orientation of the generated ions from the sample to the detector [18]. SMALDI ion microscopes and related techniques have the potential to produce high-resolution MALDI-MS images of biological tissue. However, the spatial resolution of MALDI imaging of biological tissues and single cells has thus far been limited by microprobe size, issues of sensitivity, and analyte migration during sample preparation (for reviews see [10, 12, 21].

Here, we describe an approach for MALDI-MS imaging that uses oversampling (the raster increment is smaller than the laser beam width) coupled with complete MALDI matrix ablation. Using this approach, features considerably smaller than the laser beam are imaged. The success of the technique is demonstrated by imaging a peptide standard deposited on electron microscopy (EM) calibration grids and by imaging neuropeptides that originate from single cells.

\section{Experimental}

\section{Determination of Laser Spot Size}

A saturated solution of $\alpha$-cyano-4-hydroxycinnamic acid (HCCA) in acetone was deposited onto an untreated glass cover slip to prepare a thin, dense field of matrix crystals. A VSL-337 ND-S nitrogen laser (supplied with the Voyager DE-STR mass spectrometer) focused on the sample through a single lens was fired repeatedly at specific positions, using a laser irradiation similar to what was used in image acquisition, until the matrix crystals were ablated from the glass surface. The resulting patches were optically inspected using an inverted Zeiss Axiovert 100 microscope (Carl Zeiss, Oberkochen, Germany). Regions of ablated matrix crystals appear as black patches in dark-field optical images (Figure 1). As the MS imaging approach discussed in this study concerns the complete ablation of matrix and analyte from regions of a sample, the observed size and shape of the ablated regions is taken as a reasonable measure of the effective laser beam size. With some laser positions, intervening DE grid wires can lead to curious patterns in which the effective laser beam footprint is divided into discontinuous regions (Figure 1 , left). In this study, the laser beam was positioned to produce a single continuous region of laser intensity (Figure 1, right).

\section{Coating Metal Grids with Peptides}

EM calibration grids (Electron Microscopy Science, Hatfield, PA) with a $100 \mu \mathrm{m}$ hole-to-hole spacing were used for experiments with peptide standards and were coated with solutions of $10 \mathrm{mg} / \mathrm{mL}$ HCCA dissolved in 90:10 acetone:water with an added $100 \mu \mathrm{M}$ angiotensin I (a 1297 Da peptide). EM calibration grids used for single-cell preparations were coated with a matrix so-

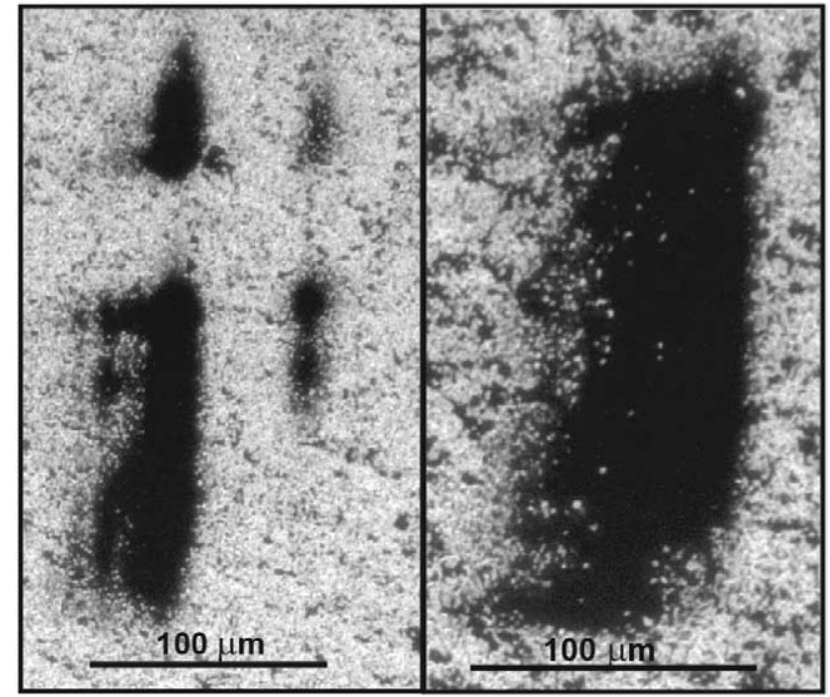

Figure 1. Dark-field optical microscope images featuring dark regions that approximate the laser beam footprint when the laser is positioned to: (left) intersect the delayed extraction (DE) grid and (right) avoid the DE grid (the right position was used for these experiments).

lution containing $10 \mathrm{mg} / \mathrm{mL}$ HCCA dissolved in 80:20 ethanol (95\%):water with $0.1 \%$ TFA and $2 \%$ acetic acid. Matrix was applied to the EM calibration grids by electrospray (ES) deposition using borosilicate glass capillary ES emitters prepared with a P80 capillary puller (Sutter Instruments, Novato, CA). A potential of $\sim 1500-2000 \mathrm{~V}$ was applied to the solution through a wire inserted into the capillary. The electrospray plume was visible as a fine mist during deposition. Grounded EM calibration grids were suspended between the ES emitter and a grounded metal plate and were effectively and rapidly coated in matrix and standards. Coating times varied from $\sim 20 \mathrm{~s}$ to $\sim 1 \mathrm{~min}$. ES emitter flow rates were typically $\sim 1 \mu \mathrm{L} / \mathrm{min}$.

\section{Cellular Preparation}

Live specimens of the marine mollusk Aplysia californica were obtained from Charles Hollahan (Santa Barbara, CA) and housed in an aerated aquarium filled with artificial seawater in our laboratory before experiments. Peptidergic neurons were isolated from the Aplysia californica central nervous system (CNS) using protocols similar to those described previously [16]. Neurons were deposited on untreated nickel EM calibration grids under the Leica dissecting microscope.

\section{Mass Spectrometry}

A MALDI-TOF Voyager DE-STR mass spectrometer (Applied Biosystems, Foster City, CA) operated in linear mode was used for all of these experiments. MS imaging was done in automatic mode with precoded translation stage coordinates that sampled in a raster pattern. In this method, the first position to be imaged 

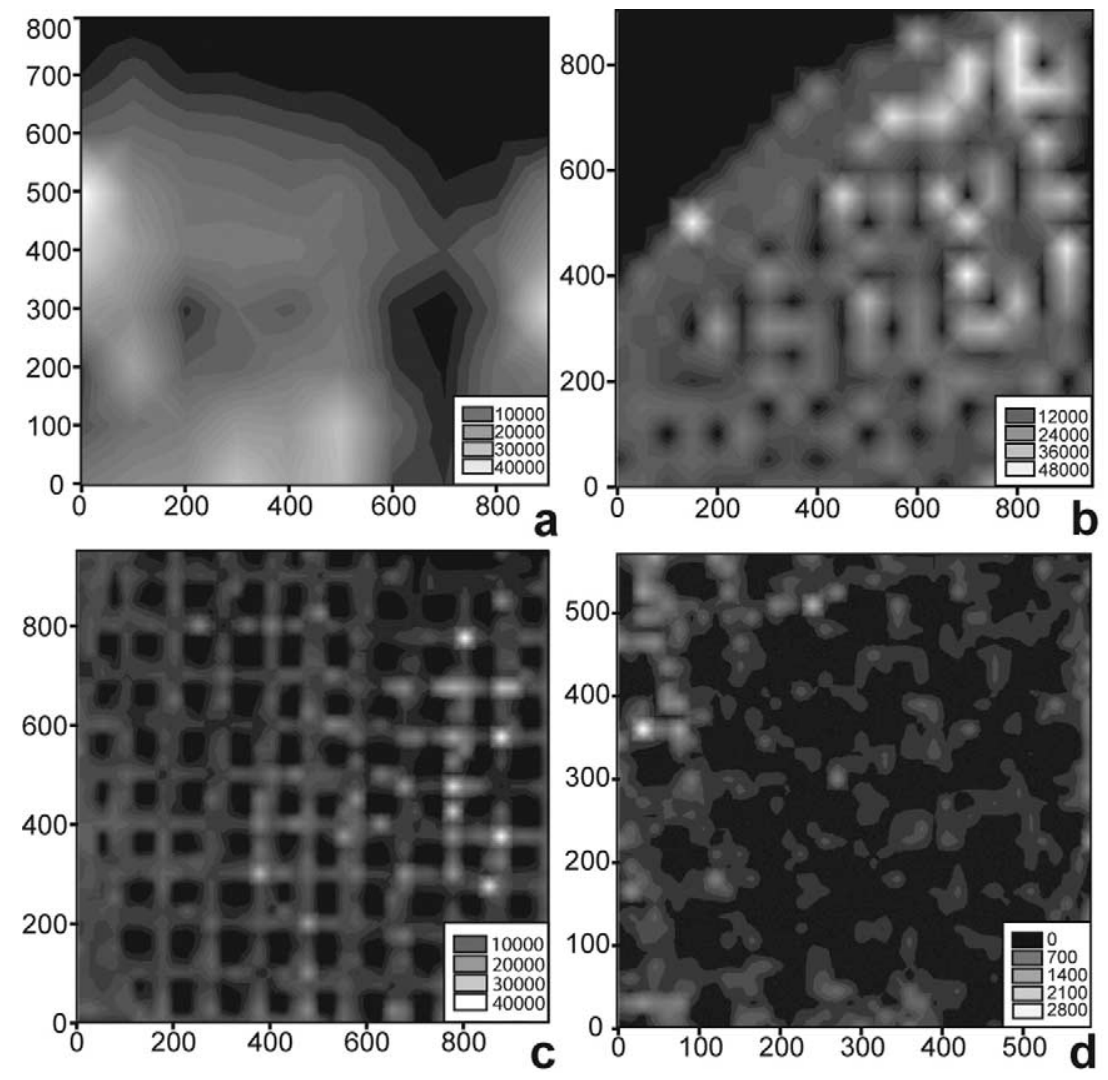

Figure 2. MALDI-MS images of the peptide angiotensin I $(\mathrm{m} / \mathrm{z}$ 1297) electrospray deposited on electron microscopy calibration grids (100 $\mu \mathrm{m}$ hole-to-hole spacing) with HCCA, using oversampling with complete sample ablation. MALDI imaging was done with (a) $100 \mu \mathrm{m}$, (b) $50 \mu \mathrm{m}$, (c) $25 \mu \mathrm{m}$, and (d) $15 \mu \mathrm{m}$ raster increments. The $\mathrm{x}$ and $\mathrm{y}$ axis of each image indicates distance in microns.

is the bottom-left corner of the sample region. The translation stage is then moved from right to left in small increments under the stationary laser beam, and a mass spectrum is collected at each position. When the bottom-right position of the imaged sample area is reached, the translation stage is moved so that the laser is directed at the left-most position of the second line in the raster. The process is repeated until the entire sample region of interest is imaged. Note that the first complete row of pixels is discarded as it records analyte signal originating from a swath the height of the entire laser beam ( $\sim 200 \mu \mathrm{m}$ in our case). The number of laser shots necessary to completely ablate matrix and analyte from the surface of the EM grids at each incremental position is experimentally determined for each sample. Complete sample ablation was accompanied by both a loss of signal (further attempts to get signal from an ablated region yielded only digitizer noise), and the appearance of a clean metallic region on the grid. Step sizes for the raster pattern were varied from 100 to 10 $\mu \mathrm{m}$ to test the limits of resolution attainable by this method. MS images of samples containing single cells were collected with raster increments of $25 \mu \mathrm{m}$.

All mass spectra for an image were collected as a single data file using the Voyager 5.1 software supplied with the mass spectrometer. Spectra were processed for baseline correction, and peak lists were extracted to prepare lists of $x-y$ position and intensity for each ion of interest using Visual Basic macros written in house. Final MS images were prepared as contour plots using SigmaPlot (Systat Software, Port Richmond, CA).

\section{Results and Discussion}

\section{Peptide Standards on Nickel Grids}

Square-holed, EM calibration grids were selected for this study and were imaged using a laser beam having dimensions of $\sim 100 \times 200 \mu \mathrm{m}$ (Figure 1, right). Features of the EM grids are considerably smaller than the laser beam size. For example, grid wires are $40 \mu \mathrm{m}$ wide and the "holes" between grid wires are $60 \mu \mathrm{m}$ on a side. An image collected with the raster increment of the translation stage set to $100 \mu \mathrm{m}$ reveals a flat, mostly featureless profile with only the rough outline of the circular EM calibration grid visible (Figure 2a). This is the expected result for images where the probe size and the raster increment are larger than the feature size.

The MS image presented in Figure 2a has the highest expected resolution based on the size of the laser beam. However, further enhancements in image resolution are possible by oversampling the surface while completely 


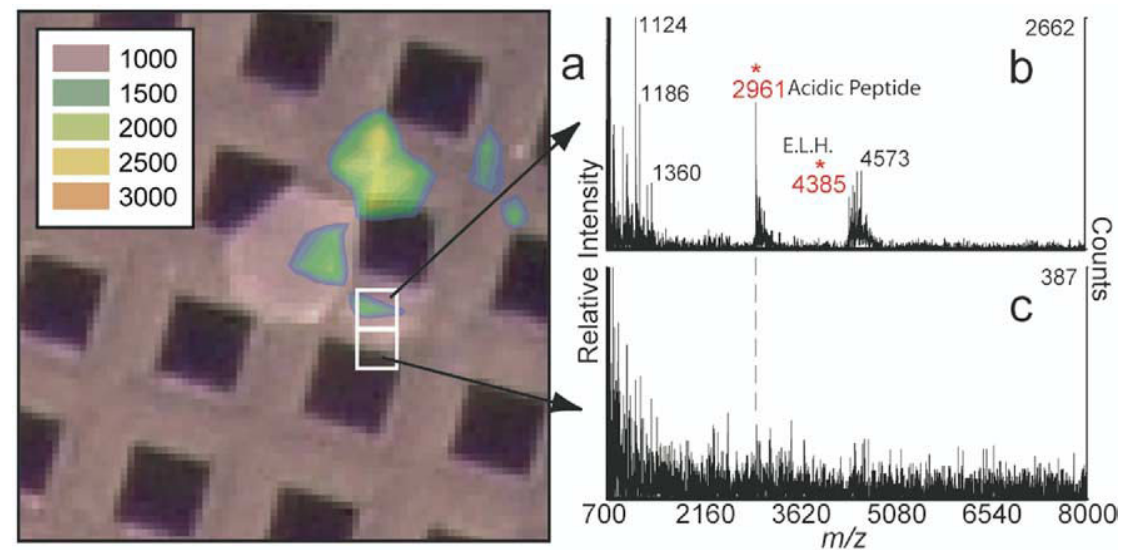

Figure 3. (a) Overlay of a MALDI-MS image of acidic peptide $(\mathrm{m} / \mathrm{z} 2961)$ acquired using the complete ablation approach and a photograph of the peptidergic Aplysia neuron, mounted on an electron microscopy calibration grid (100 $\mu \mathrm{m}$ hole-to-hole spacing), from which the image was generated. Adjacent mass spectra, each contributing a pixel to the image, show (b) high and (c) relatively low signal intensity for acidic peptide and egg-laying hormone (ELH). The abundance of the peptides of interest (indicated by asterisks) changes dramatically within a $25 \mu \mathrm{m}$ raster increment. The dashed line indicates the expected position of acidic peptide in the lower spectrum.

ablating the sample and matrix from the surface being imaged. Oversampling (acquiring signal from adjacent positions spaced by distances smaller than the sample probe) is routinely used in microprobe analysis, primarily to avoid undersampling [19]. Here, we apply oversampling in a very different way, to actually enhance spatial imaging resolution. This is done by firing the laser at each sample position until no further signal is obtained (mass spectra collected with additional laser shots yield only digitizer noise). If the sample translation stage is then moved, all signal intensity from the new sample position will arise from the overlap of fresh sample and the portion of the laser beam with sufficient laser fluence to produce ion signal. This is true even if the increment of the translation stage is significantly less than the width of the laser beam. In essence, given complete ablation, the sampling is done with a small region at the leading edge of the laser beam.

Oversampling with complete sample ablation provides a significant resolution enhancement, as demonstrated in Figure $2 b$ and $c$, where the translation stage raster step size has been reduced to 50 and $25 \mu \mathrm{m}$ and the analyte and matrix are completely ablated at each sample position. As the latter raster increment is well below the smallest feature size of the subject being imaged (in this case, the wire width of $40 \mu \mathrm{m}$ ), all features of the grid are clearly resolved, a remarkable feat when using a probe with $\sim 100 \times 200 \mu \mathrm{m}$ dimensions.

Further decreases in the raster increment lead to a gradual reduction in image quality under the conditions of our experiment (for example, Figure 2d). This is likely attributable to a combination of factors, including irregularities in translation stage movement and matrix crystal size, the fall off of laser fluence at the edge of the beam [22], and a general decrease in available analyte at smaller raster increments.

\section{MS Images of Cellular Peptides and Single Cells}

Aplysia californica peptidergic neurons (typically 80 to $100 \mu \mathrm{m}$ in diameter) were deposited on untreated nickel EM calibration grids and coated with HCCA using electrospray. These cells were selected for both their large size and well-characterized neuropeptide compliments. The deposition of these neurons onto nickel EM grids and the removal of extracellular solution tend to result in partial or complete cell lysis. The example shown is of a cell that remained intact by visual inspection throughout the preparation and matrix application. Still, MALDI-MS imaging revealed that neuropeptides had spread from the cell onto the surrounding grid wires. This is readily apparent in an overlay of a chemical image of Aplysia acidic peptide $(2961 \mathrm{~m} / \mathrm{z})$ and a photograph of the EM grid containing the cells (Figure 3a). Spectra from adjacent $25 \mu \mathrm{m}$ pixels show dramatic abundance variations for the analyte of interest (Figure $3 b$ and c), demonstrating that real analytical distributions smaller than the size of the laser beam can be imaged, provided that the analyte can be effectively mixed with the matrix and exposed to the laser beam.

\section{Methodological Details of Oversampling with Complete Ablation}

Given a typical commercial MALDI instrument capable of operation in unattended, automatic mode, the two essential experimental variables that must be controlled are the correct matrix deposition and the number of laser shots necessary for "complete sample ablation." MALDI matrix must be applied sufficiently wet so that the analyte previously in the sample is incorporated into the matrix, but must dry rapidly enough to minimize analyte migration [10, 20], and must form a 
uniform layer of preferably small crystals. To use oversampling with complete ablation, the layer of applied matrix must be thin enough so that complete matrix ablation occurs within a reasonable number of laser shots. In the experiments presented here, a maximum of 80 pulses/scan was used, with most of the images acquired using 40 pulses/scan or less. The use of this number of laser pulses for an analysis is not prohibitive compared with the 30 to 250 shots typically used in conventional high-resolution MALDI-MS imaging [10].

Determining the number of laser pulses necessary for complete ablation is also important. If insufficient laser pulses are used, some matrix will remain at each translation stage position after moving the stage, and imaging resolution will decrease significantly. In contrast, a large excess of laser shots over what is needed for complete sample ablation will unnecessarily average noise into each mass spectrum, thereby decreasing the dynamic range of the image and possibly losing information about positions in the sample that have a low abundance of the analytes of interest. In general, imaging sensitivity was improved by using slightly less $(75-80 \%)$ of the laser pulses necessary for complete ablation. While the use of incomplete ablation decreases the resolution of the image below what would otherwise be achievable with the raster increment, it leads to a higher $\mathrm{S} / \mathrm{N}$ for analyte signals as less noise is averaged into each spectrum, thus representing a compromise between sensitivity and resolution. However, the last 10 to $20 \%$ of the laser pulses required for complete ablation contribute less analyte signal intensity than the earlier laser pulses, diminishing the detrimental effects of imaging resolution that might otherwise be expected. Nonetheless, the use of an insufficient number of laser pulses results in a somewhat smeared-out grid appearance.

\section{Conclusions}

This work describes a new approach for MALDI-MS imaging, oversampling with complete sample ablation, which enables the acquisition of data to develop chemical images with a resolution higher than the limitations imposed by the size of the laser beam size. This technique should, in principle, allow any commercial MALDI mass spectrometer equipped with reasonably accurate and reproducible translation stage motion to acquire moderate to high-resolution MS images without the need for any modifications to be made to the laser optics. This approach has been characterized using both peptide standards and peptides originating from peptidergic neurons. The use of oversampling coupled with complete sample ablation for MS imaging may prove advantageous for research groups using facility or multiuse instruments that service a variety of both imaging and nonimaging research projects, as no instrument optical alignments or adjustments are necessary between samples. When used with instruments that have already been modified to produce smaller laser beam sizes, this tech- nique may provide further enhancements to their attainable imaging spatial resolution.

The capability to clearly image features less than $40 \mu \mathrm{m}$ in size (with a $25 \mu \mathrm{m}$ raster increment), allows commercial MALDI instruments with large laser beams to image with a resolution similar to the highest resolution indicated in many of the published experiments [8, 12-16, 19]. Although further improvements in MALDI-MS imaging resolution are certainly conceivable by applying this or other methods, and have already been proven using standards or inorganic samples [17, 18], MALDI-MS imaging of complex organic samples, such as biological tissue, with a resolution beyond what is described in these experiments, is not currently limited by instrumentation but rather by available techniques of matrix application, analyte extraction, and sensitivity. The next hurdle for MALDI-MS imaging is the application of matrix in a manner where the analyte of interest is quantitatively (or at least efficiently) extracted from biological tissue and incorporated into MALDI matrix with a suitable matrix/analyte ratio, while maintaining a high degree of spatial information. Such a matrix application technique has yet to be demonstrated.

\section{Acknowledgments}

The authors thank Mr. Cory Scanlan for initial assistance in EM disk preparation, and Mr. Eric B. Monroe for assistance in figure preparation. JJ gratefully acknowledges support through an NRSA traineeship sponsored by the NIAAA 1F32 AA014459. This work was supported by NIH through DA14879 and the UIUC Neuroproteomics Center on Cell-Cell Signaling through grant P30 DA018310.

\section{References}

1. Karas, M.; Bachmann, D.; Bahr, U.; Hillenkamp, F. MatrixAssisted Ultraviolet-Laser Desorption of Nonvolatile Compounds. Int. J. Mass Spectrom. Ion Processes 1987, 78, 53-68.

2. Karas, M.; Bahr, U.; Ingendoh, A.; Hillenkamp, F. Laser Desorption Ionization Mass-Spectrometry of Proteins of Mass 100,000 to 250,000 Dalton. Angew. Chem. Int. Ed. Engl. 1989, 28 (6), 760-761.

3. Yamashita, M.; Fenn, J. B. Electrospray Ion-Source-Another Variation on the Free-Jet Theme. J. Phys. Chem. 1984, 88(20), 4451-4459.

4. Gusev, A. I.; Vasseur, O. J.; Proctor, A.; Sharkey, A. G.; Hercules, D. M. Imaging of Thin-Layer Chromatograms Using Matrix/ Assisted Laser Desorption/Ionization Mass-Spectrometry. Anal. Chem. 1995, 67(24), 4565-4570.

5. Spengler, B.; Hubert, M.; Kaufmann, R. MALDI Ion Imaging and Biological Ion Imaging with a New Scanning UV-Laser Microprobe. Proceedings of the 42nd ASMS Conference on Mass Spectrometry and Allied Topics; Chicago, IL, May, 1994.

6. Caprioli, R. M.; Farmer, T. B.; Gile, J. Molecular Imaging of Biological Samples: Localization of Peptides and Proteins Using MALDI-TOF MS. Anal. Chem. 1997, 69(23), 4751-4760.

7. Zhang, H.; Stoeckli, M.; Andren, P. E.; Caprioli, R. M. Combining Solid-Phase Preconcentration, Capillary Electrophoresis and Off-Line Matrix-Assisted Laser Desorption/Ionization Mass Spectrometry: Intracerebral Metabolic Processing of Peptide E in Vivo. J. Mass Spectrom. 1999, 34(4), 377-383. 
8. Stoeckli, M.; Chaurand, P.; Hallahan, D. E.; Caprioli, R. M. Imaging Mass Spectrometry: A New Technology for the Analysis of Protein Expression in Mammalian Tissues. Nat. Med. 2001, 7(4), 493-496.

9. Reyzer, M. L.; Hsieh, Y. S.; Ng, K.; Korfmacher, W. A.; Caprioli, R. M. Direct Analysis of Drug Candidates in Tissue by Matrix-Assisted Laser Desorption/Ionization Mass Spectrometry. J. Mass Spectrom. 2003, 38(10), 1081-1092.

10. Schwartz, S. A.; Reyzer, M. L.; Caprioli, R. M. Direct Tissue Analysis Using Matrix-Assisted Laser Desorption/Ionization Mass Spectrometry: Practical Aspects of Sample Preparation. J. Mass Spectrom. 2003, 38(7), 699-708.

11. Yanagisawa, K.; Shyr, Y.; Xu, B. G. J.; Massion, P. P.; Larsen, P. H.; White, B. C.; Roberts, J. R.; Edgerton, M.; Gonzalez, A.; Nadaf, S.; Moore, J. H.; Caprioli, R. M.; Carbone, D. P. Proteomic Patterns of Tumor Subsets in Non-Small-Cell Lung Cancer. Lancet 2003, 362(9382), 433-439.

12. Chaurand, P.; Schwartz, S. A.; Billheimer, D.; Xu, B. G. J.; Crecelius, A.; Caprioli, R. M. Integrating Histology and Imaging Mass Spectrometry. Anal. Chem. 2004, 76(4), 1145-1155.

13. Garden, R. W.; Sweedler, J. V. Heterogeneity Within MALDI Samples as Revealed by Mass Spectrometric Imaging. Anal. Chem. 2000, 72(1), 30-36.

14. Stoeckli, M.; Staab, D.; Staufenbiel, M.; Wiederhold, K. H.; Signor, L. Molecular Imaging of Amyloid $\beta$-Peptides in Mouse Brain Sections Using Mass Spectrometry. Anal. Biochem. 2002, 311(1), 33-39.

15. Kruse, R.; Sweedler, J. V. Spatial Profiling Invertebrate Ganglia Using MALDI MS. J. Am. Soc. Mass Spectrom. 2003, 14(7), 752-759.
16. Rubakhin, S. S.; Greenough, W. T.; Sweedler, J. V. Spatial Profiling with MALDI MS: Distribution of Neuropeptides Within Single Neurons. Anal. Chem. 2003, 75(20), 5374-5380.

17. Spengler, B.; Hubert, M. Scanning Microprobe MatrixAssisted Laser Desorption Ionization (SMALDI) Mass Spectrometry: Instrumentation for Submicrometer Resolved LDI and MALDI Surface Analysis. J. Am. Soc. Mass Spectrom. 2002, 13(6), 735-748.

18. Luxembourg, S. L.; Mize, T. H.; McDonnel, L. A.; Heeren, R. M. A. High-Spatial Resolution. Mass Spectrometric Imaging of Peptide and Protein Distributions on a Surface. Anal. Chem. 2004, 76, 5339-5344.

19. Todd, P. J.; Schaaff, T. G.; Chaurand, P.; Caprioli, R. M. Organic Ion Imaging of Biological Tissue with Secondary Ion Mass Spectrometry and Matrix-Assisted Laser Desorption/ Ionization. J. Mass Spectrom. 2001, 36(4), 355-369.

20. Chaurand, P.; Caprioli, R. M. Direct Profiling and Imaging of Peptides and Proteins from Mammalian Cells and Tissue Sections by Mass Spectrometry. Electrophoresis 2002, 23(18), 3125-3135.

21. Chaurand, P.; Schwartz, S. A.; Caprioli, R. M. Assessing Protein Patterns in Disease Using Imaging Mass Spectrometry. J. Proteome Res. 2004, 3(2), 245-252.

22. Dreisewerd, K.; Schurenberg, M.; Karas, M.; Hillenkamp, F. Influence of the Laser Intensity and Spot Size on the Desorption of Molecules and Ions in Matrix-Assisted LaserDesorption Ionization with a Uniform Beam Profile. Int. J. Mass Spectrom. Ion Processes 1995, 141(2), 127-148. 